\title{
Asociación entre las características empresariales y la divulgación sobre Capital Intelectual: Un estudio de las empresas del Ibex 35
}

\author{
Francisca TEJEdo ROMERO a ${ }^{\text {, ESTEBAN AlFARO CORTÉS }}{ }^{\text {a }}$ \\ a Universidad de Castilla-La Mancha, Facultad CC.EE., Campus Universitario, Plaza de la Univer- \\ sidad,2,02071 Albacete, España.E-mail: Francisca.Tejedo@uclm.es, Esteban.Alfaro@uclm.es
}

\section{RESUMEN}

El propósito de este trabajo es conocer si las empresas del Ibex 35 están divulgando información sobre el Capital Intelectual y determinar aquellas características empresariales que pueden condicionarlas a un mayor suministro de información. Para ello se analizan 115 informes anuales correspondientes a las 23 empresas de la muestra para un periodo de cinco años. La metodología utilizada para obtener la información fue el análisis de contenidos, y se llevó a cabo un análisis descriptivo y un análisis multivariante de regresión lineal. Los resultados muestran que los niveles de información del Capital Intelectual no son muy elevados. No obstante, el tamaño, el endeudamiento, el sector y el ratio market to book inciden en la cantidad de información del Capital Intelectual que se divulga.

Palabras clave: Capital Intelectual, divulgación, análisis de contenido, informes anuales.

\section{Association between Business Characteristics and Intellectual Capital Disclosure: A Study of Companies in the Ibex 35}

\begin{abstract}
This study aims to analyze the specific characteristics which lead some Ibex 35 Spanish companies to disclose higher levels of information about their Intellectual Capital. For this purpose, we use a sample of 115 corporate annual reports, which corresponds to the information published by 23 companies in the Ibex 35 during a period of 5 years. The methodology used to obtain the sample was content analysis, and we carry out a descriptive analysis and a multivariate linear regression analysis. The results show low levels of Intellectual Capital disclosure. However, the size, the leverage, the industry and the market to book affect the provision of this information.
\end{abstract}

Keywords: Intellectual Capital, Disclosure, Content Analysis, Annual Reports.

Clasificación JEL: M14, M41

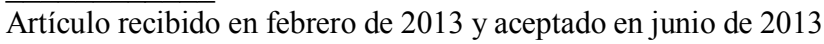

Artículo disponible en versión electrónica en la página www.revista-eea.net, ref. ə-32103 


\section{INTRODUCCIÓN}

El creciente auge que han adquirido los intangibles, motivado por la competitividad, la globalización del comercio, la desregularización de los mercados y los cambios tecnológicos (FASB, 2001a; Lev, 2003), ha provocado que las empresas cambien su manera de actuar. Pasando de un enfoque a corto plazo y preocupado por la gestión de sus activos tangibles, hacia un enfoque a largo plazo (Pizarro et al., 2011) dirigido a invertir en intangibles y generar conocimiento (Capital Intelectual). Estos últimos se han convertido en los principales factores claves del éxito en las empresas (Bueno et al., 2008; Cañibano y Sánchez, 2008; Ramírez, 2010; Yi y Davey, 2010).

Pero la falta de consenso, en cuanto a la denominación de los intangibles, alcanza tanto al concepto como a la naturaleza y clasificación de los mismos (Simó y Sallán, 2008). Así, se utilizan los términos de conocimiento, activos del conocimiento, intangible, activos intangibles, recursos intangibles y Capital Intelectual, indistintamente, para referirse al mismo fenómeno (Pulido, 2008). Aunque el término de Capital Intelectual es el más utilizado por la investigación académica contemporánea (Joshi et al., 2012), y será el que se utilice en este trabajo.

La Comisión Europea (2006) considera que el Capital Intelectual es la combinación de los recursos y actividades humanas, organizativas y relacionales de una entidad y por tanto, es el conocimiento, competencias, experiencia y habilidades de los trabajadores (Capital Humano); las actividades de investigación y desarrollo, las rutinas organizativas, procedimientos, sistemas, bases de datos y derechos de propiedad intelectual (Capital Estructural); así como, los recursos ligados a las relaciones exteriores con clientes, proveedores y socios de $\mathrm{I}+\mathrm{D}$ (Capital Relacional).

La mayoría de los investigadores coinciden en afirmar que el Capital Intelectual aumenta considerablemente el valor de una empresa (Abeysekera, 2007; Ferreira et al., 2012; Striukova et al., 2008; Wagiciengo y Belal, 2012). Sin embargo, su valor se mantiene oculto y no es reconocido en los balances de las empresas (Lev, 2003), debido a las dificultades que plantea su reconocimiento, medición y valoración (Beattie, 2005; Brennan, 2001). Lo que da lugar a la diferencia existente entre el valor contable y el valor de mercado en las empresas $^{1}$. Ello implica una pérdida de utilidad de la información suministrada por las empresas para la toma de decisiones de sus grupos de interés (Collins et al., 1997; Francis y Schipper, 1999; García-Ayuso et al., 1998; Lev y Zarowin, 1999; Wallman, 1995).

\footnotetext{
${ }^{1}$ Algunos autores consideran que esa diferencia no sólo puede ser atribuida al Capital Intelectual, dado que los mercados financieros no son eficientes y, además, los activos del balance están valorados al coste histórico (Gu y Lev, 2001; Upton, 2001; García-Ayuso, 2003).
} 
Esta falta de reconocimiento del Capital Intelectual en el balance, está motivada por el incumplimiento de los criterios de reconocimiento y medición, establecidos por los organismos contables (FASB, 2001b; IASB, 2004; PGC, 2007), para poder ser considerado como un activo intangible. Como resultado, una parte significativa de los activos de las empresas no pueden ser identificados en sus estados financieros y, por tanto, los inversores no reciben suficiente información para tomar decisiones eficientes (Abeysekera, 2007), al igual que el resto de grupos de interés.

En este sentido, Abeysekera (2007) señala que, los reguladores contables han ignorado la necesidad de incorporar información relativa al Capital Intelectual como parte de los estados financieros. Por eso, las empresas están interesadas en ofrecer información voluntaria (Bonsón y Escobar, 2004; Bukh y Johanson, 2003; Castilla y Gallardo, 2008) con la finalidad de reducir los problemas que generan las asimetrías de información y mejorar la transparencia entre los grupos de interés (Ramírez et al., 2011; Vergauwen et al., 2007; Yi y Davey, 2010).

Son numerosos los estudios que han tratado de analizar si las empresas están suministrando información voluntaria sobre su Capital Intelectual (Abeysekera, 2006; Abeysekera y Guthrie, 2005; Beaulieu et al., 2002; Bontis, 2003; Bozzolan et al., 2003; Branco et al., 2011; Brennan, 2001; Bukh et al., 2005; Campbell y Abdul Rahman, 2010; Cerbioni y Parbonetti, 2007; Chander y Mehra, 2011; Ferreira et al., 2012; Firer y Williams, 2005; Gandía Cabedo, 2002; García-Meca et al., 2005; Guthrie y Petty, 2000; Guthrie et al., 2004; Joshi et al., 2012; Li et al., 2008; Monclús et al., 2006; Oliveira et al., 2010; Oliveras et al., 2008; Singh y Kansal, 2011; Steenkamp, 2007; Whiting y Miller, 2008; Whiting y Woodcock, 2011; Williams, 2001; Yi y Davey, 2010). Algunos de estos trabajos se han basado en los postulados de la teoría de la agencia, teoría de la señal, teoría de la legitimidad, teoría de los stakeholders y en la teoría de recursos y capacidades para explicar los motivos por los cuales las empresas suministran información voluntaria.

Sin embargo, y bajo el marco de las anteriores teorías, existen una serie de características empresariales que han sido discutidas en trabajos previos (Bozzolan et al., 2003; Branco et al., 2011; Ferreira et al., 2012; Firer y Williams, 2005; García-Meca et al., 2005; Joshi et al., 2012; Striukova et al., 2008; White et al., 2007; Whiting y Miller, 2008; Whiting y Woodcock, 2011; Williams, 2001), como pueden ser el tamaño, el nivel de endeudamiento, la rentabilidad, el sector, entre otras, que pueden inducir a las empresas a divulgar más información que otras.

Esto plantea la necesidad de establecer un marco común y generalmente aceptado para la presentación de información relativa al Capital Intelectual, estos son los principales motivos que justifican la necesidad de abordar nuestro 
trabajo. Así, en el ámbito de las empresas españolas, el objetivo de este estudio es conocer si las empresas del Ibex 35 están informando de manera voluntaria sobre su Capital Intelectual e identificar aquellas características empresariales que pueden impulsarlas hacia prácticas de revelación de información voluntaria del Capital Intelectual.

A este fin, se han analizado 115 informes anuales, correspondientes a 23 empresas incluidas en el Ibex 35 durante los 5 años objeto de estudio. Se ha empleado la metodología del análisis de contenidos que ha permitido codificar la información (Krippendorff, 1997; Weber, 1990). Posteriormente, se han elaborado índices de divulgación para cuantificar la información (Marston y Shrives, 1991). Las técnicas estadísticas empleadas han sido el análisis descriptivo y multivariante de regresión lineal.

Los resultados han permitido confirmar que el tamaño, nivel de endeudamiento, sector industrial y el ratio market to book inciden en el suministro de información voluntaria sobre el Capital Intelectual. No obstante, aunque estamos inmersos en la Era del Conocimiento, los niveles de información suministrados por las empresas españolas del Ibex 35 relativos al Capital Intelectual no son muy elevados.

Así, a pesar de la estricta normativa contable respecto al reflejo del Capital Intelectual en los estados financieros de las empresas, este trabajo puede ayudar a organismos e instituciones, públicos y privados, a intentar elaborar unas guías o directrices respecto a la presentación de información voluntaria del Capital Intelectual, o bien, a desarrollar mejores normativas contables, ya que se ha evidenciado que con la intención de minorar la brecha existente entre el valor de mercado y el contable, las empresas sí están suministrando información adicional a sus grupos de interés con la finalidad de que éstos tomen decisiones eficientes.

Esto puede ser especialmente relevante para el resto del tejido empresarial en cuanto a mejorar los niveles y la cantidad de información suministrada del Capital Intelectual, puesto que las empresas (bajo los postulados de diferentes teorías: agencia, señal, stakeholders, legitimidad y recursos y capacidades) son conscientes de la importancia que tiene el Capital Intelectual para sus grupos de interés y se da a conocer sólo por algunas empresas.

\section{LITERATURA PREVIA Y DESARROLLO DE HIPÓTESIS}

\subsection{Teorías y estudios de divulgación de Capital Intelectual}

Desde un punto de vista teórico, los incentivos por los cuales las empresas divulgan voluntariamente información del Capital Intelectual pueden ser explicados en términos de una gran variedad de teorías. Algunos estudios toman como referencia el marco de la teoría de la agencia (Firer y Williams, 2005; 
García-Meca et al., 2005), la teoría de la señal (García-Meca et al., 2005; Guthrie y Petty, 2000; Oliveras et al., 2008), la teoría de los stakeholders (Abeysekera y Guthrie, 2005; Bozzolan et al., 2003; Whiting y Miller, 2008; Yi y Davey, 2010), la teoría de la legitimidad (Guthrie et al., 2004; Oliveira et al., 2010) y la teoría de recursos y capacidades (Abeysekera, 2006; Oliveira et al., 2010).

En base a los postulados de la teoría de la agencia (Jensen y Meckling, 1976), las asimetrías de información generan un problema de agencia entre el principal (accionistas/propietarios) y el agente (directivos/gerentes) y, por tanto, unos costes (los costes de agencia). En este sentido, la divulgación de Capital Intelectual puede ser una vía importante para reducir las asimetrías de información existentes entre los gestores y los accionistas y, en consecuencia, los costes de agencia.

La teoría de la señal (Ross, 1979), muy relacionada con la anterior, postula que las empresas a través de la información voluntaria divulgada están enviando señales al mercado sobre determinadas características no observables de las empresas (Giner, 1997). Así, las empresas a través de la información voluntaria del Capital Intelectual pueden enviar señales al mercado acerca de las principales fuentes futuras de creación de riqueza.

Conforme al enfoque de la teoría de los stakeholders (Freeman, 1984), el éxito y supervivencia a largo plazo de la empresa se encuentran sometidos a la aprobación de los grupos de interés. En este sentido, la divulgación voluntaria de información puede ser el medio para negociar la relación empresa-stakeholders. Abeysekera y Guthrie (2005) sostienen que a la empresa le interesa suministrar a los grupos de interés información relativa al Capital Intelectual con el fin de aumentar el valor percibido de la misma.

La teoría de la legitimidad sugiere que existe un contrato social entre la empresa y la sociedad en la que opera (Shocker y Sethi, 1973). Así, para que las empresas sean legitimadas por la sociedad es necesario que comuniquen información de carácter voluntario. Por tanto, las empresas pueden estar interesadas en suministrar información sobre el Capital Intelectual por razones de legitimidad, ya que aquellas empresas intensivas en recursos intangibles (con altos niveles de Capital Intelectual) están más interesadas a revelar dicha información debido a que no pueden legitimar su situación a través de los estados contables tradicionales.

Desde la perspectiva de la teoría de recursos y capacidades, el éxito y supervivencia de la empresa dependen en gran medida de los recursos y capacidades de los que dispone. Los recursos son los activos que posee la empresa, que pueden ser tangibles o intangibles, y las capacidades son los conocimientos y habilidades acumulados a través de rutinas empresariales. Dentro de los recursos, el Capital Intelectual es considerado el más importante por su potencial estratégico 
en generar ventajas competitivas en las empresas (Hall, 1992). Por eso, debido a la importancia estratégica del Capital Intelectual, muchas empresas están interesadas en informar de manera voluntaria con el fin de señalar su ventaja competitiva (Sonnier, 2008) y así, poder conseguir el apoyo de los inversores en el mercado de capitales.

En cuanto a la investigación empírica, destacan los trabajos que analizan la información sobre Capital Intelectual suministrada por las empresas y los que pretenden identificar aquellos factores o características empresariales que determinan el suministro de información. Ahora bien, a pesar de la existencia de una variedad de marcos conceptuales que han sido utilizados para identificar, clasificar y definir al Capital Intelectual (Brooking, 1997; Euroforum, 1998; Kaplan y Norton, 1992; Sveiby, 1997), no se ha normalizado ninguno de ellos en todo el mundo. Sin embargo, la inmensa mayoría de estudios de divulgación se han basado en el marco inicial de Sveiby (1997), que posteriormente fue modificado por Guthrie y Petty (2000) y replicado y modificado por numerosos investigadores (Abeysekera y Guthrie, 2005; April et al., 2003; Bozzolan et al., 2003; Brennan, 2001; Ensslin y De Carvalho, 2007; Ferreira et al., 2012; Gan et al., 2008; Goh y Lim, 2004; Guthrie et al., 2006; Oliveira et al., 2010; Oliveras et al., 2008; Steenkamp, 2007; Vandemaele et al., 2005).

La mayoría de los estudios de divulgación del Capital Intelectual se han centrado en analizar la cantidad de información suministrada por parte de las empresas, normalmente las incluidas en índices de cotización de un país específico y un periodo de tiempo corto, habitualmente de un año. Así, destacan los trabajos realizados en: Australia (Guthrie y Petty, 2000; Sujan y Abeysekera, 2007; White et al., 2007; Whiting y Woodcock, 2011), Irlanda (Brennan, 2001), Italia (Bozzolan et al., 2003), Sudáfrica (April et al., 2003; Wagiciengo y Belal, 2012), Malasia (Goh y Lim, 2004), Sri Lanka (Abeysekera y Guthrie, 2005), Hong Kong (Petty y Cuganesan, 2005), España (Gandía Cabedo, 2002; GarcíaMeca et al., 2005; Monclús et al., 2006; Oliveras et al., 2008), Brasil (Ensslin y De Carvalho, 2007), Nueva Zelanda (Steenkamp, 2007; Whiting y Miller, 2008; Wong y Gardner, 2005), Portugal (Branco et al., 2011; Ferreira et al., 2012; Oliveira et al., 2010), Reino Unido (Striukova et al., 2008), India (Chander y Mehra, 2011; Singh y Kansal, 2011), China (Yi y Davey, 2010), Países Bajos, Suecia y Reino Unido (Vandemaele et al., 2005), China y Australia (Joshi et al., 2012), entre otros. $Y$ en general, los resultados obtenidos indican que los niveles de divulgación del Capital Intelectual son bajos en todo el mundo, siendo la categoría del Capital Relacional la más divulgada, seguida del Capital Estructural y el Capital Humano el menos divulgado (Whiting y Miller, 2008).

Paralelamente, muchos de los trabajos anteriormente citados se han complementado con un análisis de aquellas características empresariales que están asociadas al mayor o menor suministro de información del Capital Intelectual 
(Bozzolan et al., 2003; Branco et al., 2011; Ferreira et al., 2012; Firer y Williams, 2005; García-Meca et al., 2005; Joshi et al., 2012; Striukova et al., 2008; White et al., 2007; Whiting y Miller, 2008; Whiting y Woodcock, 2011; Williams, 2001).

\subsection{Desarrollo de hipótesis}

En base a las consideraciones teóricas y la investigación empírica previa descrita anteriormente, a continuación se desarrollan una serie de hipótesis que relacionan las características empresariales con las prácticas de divulgación de Capital Intelectual en España.

\subsubsection{Tamaño}

Hay una serie de argumentos que se pueden utilizar para relacionar el tamaño y la divulgación de información sobre el Capital Intelectual. Esta relación puede ser explicada en base a la teoría de la agencia, señal, legitimidad y stakeholders.

Desde el enfoque de la teoría de la agencia, son las empresas de gran tamaño las que suministran más cantidad de información. En este sentido, Giner (1997) afirma que, en las grandes empresas es donde surgen los mayores conflictos de intereses entre las partes y, por tanto, tienen mayores costes de agencia. Por eso, una manera de reducir esos costes es mediante el suministro de información voluntaria. Además, son las grandes empresas las que más señales envían al mercado para reducir las asimetrías de información que se producen $\mathrm{y}$, por tanto, las que más información sobre el Capital Intelectual suministran (Bozzolan et al., 2003).

Otros autores como Haniffa y Cooke (2005) y Patten (1991) se apoyan en la teoría de la legitimidad para justificar la relación positiva existente entre el tamaño y el suministro de información adicional. Son las grandes empresas las que más información suministran puesto que están más expuestas al público y tienen que legitimar sus actividades (Belkaoui y Karpik, 1989). Por otro lado, y bajo los postulados de la teoría de los stakeholders, las empresas de gran tamaño son las que tienen un mayor número de interesados en la información voluntaria, y pueden ser las que estén más interesadas en la información del Capital Intelectual.

Los trabajos de Bozzolan et al., (2003), Eng y Mak (2003), García-Meca et al., (2005), Haniffa y Cooke (2005), Barako et al., (2006), Guthrie et al., (2006, 2007), Tagesson et al., (2009), Branco et al., (2011) y Ferreira et al., (2012) encuentran una relación positiva entre la divulgación de información de Capital Intelectual y el tamaño de la empresa. Así, se formula la siguiente hipótesis a corroborar: 
- H1: El tamaño influye de forma positiva en la divulgación de información de Capital Intelectual.

\subsubsection{Sector}

Se ha evidenciado que las empresas intensivas en Capital Intelectual divulgan más información que aquellas que poseen menos recursos intangibles. Desde un punto de vista teórico, esta relación ha sido explicada bajo los postulados de la teoría de la señal y la teoría de los stakeholders.

Bajo el enfoque de la teoría de la señal, las empresas más intensivas en Capital Intelectual suministran más información con la finalidad de enviar señales a los inversores, tanto actuales como potenciales, del verdadero valor de la empresa ya que éste no puede figurar en los estados financieros presentados por las empresas (Gandía Cabedo, 2003).

También se ha explicado desde la teoría de los stakeholders (Vergauwen et al., 2007) al considerar que, aunque las empresas no están obligadas a divulgar información voluntaria sobre el Capital Intelectual, aquellas que son intensivas en Capital Intelectual les interesará revelar a los grupos de interés mucha más información con el fin de aumentar el valor percibido de la misma. Entre los estudios que apoyan esta hipótesis se encuentran los trabajos de Bozzolan et al. (2003), Vergauwen et al., (2007), Sujan y Abeysekera (2007), Li et al., (2008), Striukova et al., (2008), Whiting y Woodcock (2011) y Ferreira et al., (2012). Por tanto, se pretende contrastar la hipótesis siguiente:

- H2: El sector productivo influye de manera significativa en la divulgación de información de Capital Intelectual.

\subsubsection{Rentabilidad}

La relación existente entre el nivel de divulgación de información del Capital Intelectual y la rentabilidad, ha sido defendida bajo los marcos de la teoría de la agencia, la teoría de la señal, la teoría de los stakeholders y la teoría de recursos y capacidades.

En este sentido y, con la finalidad de reducir el conflicto de intereses entre la dirección (gestores) y los accionistas, altas tasas de rentabilidad incentivan a la dirección a suministrar información voluntaria del Capital Intelectual con la finalidad de probar la buena marcha de la empresa, justificando de esta manera la necesidad de una continuidad en el puesto de trabajo y una mejora de sus remuneraciones (Singhvi y Desai, 1971). Igualmente, la dirección emite señales al mercado que identifiquen la buena situación que presenta la empresa (Giner, 1997).

Además, desde el punto de vista de la Teoría de los Stakeholders y de los Recursos y Capacidades, las empresas más rentables divulgan más información 
del Capital Intelectual debido a que esa rentabilidad puede ser fruto de la continua inversión en intangibles.

El estudio realizado por Li et al., (2008) en las empresas cotizadas de Reino Unido confirma que las empresas más rentables son las que mayores cantidad de información sobre el Capital Intelectual suministran. Por tanto, la tercera hipótesis a considerar será:

- H3: La rentabilidad influye de manera positiva en la divulgación de información de Capital Intelectual.

\subsubsection{Endeudamiento}

La teoría de la agencia es utilizada para explicar la influencia que tiene el nivel de endeudamiento en el suministro de información voluntaria del Capital Intelectual, ya que cuanto mayores sean los niveles de endeudamiento mayores serán los conflictos entre los acreedores y los accionistas y, por tanto, los costes de agencia (Jensen y Meckling, 1976). Así, con la finalidad de reducir los costes de agencia y el conflicto de intereses existentes, las empresas proporcionarán más información relativa al Capital Intelectual con la finalidad de asegurar a los acreedores que tiene capacidad de devolver sus deudas porque poseen recursos intangibles que le permiten obtener ventajas competitivas. En este sentido los trabajos realizados por Williams (2001) y White et al., (2007) encuentran una relación positiva. En virtud de estos planteamientos, se propone la hipótesis cuarta:

- H4: El nivel de endeudamiento influye de manera positiva en la divulgación de información de Capital Intelectual.

\subsubsection{Market to Book}

Los estudios previos han argumentado que una mayor divulgación de información relativa al Capital Intelectual podría reducir la brecha existente entre el valor de mercado y el valor en libros (Brennan, 2001; Sveiby, 2000). Esta relación se apoya tanto en la teoría de los stakeholders y la teoría de recursos y capacidades. Así, una empresa con un alto valor en el ratio market to book tiene un mayor incentivo para divulgar voluntariamente información sobre el Capital Intelectual, con el fin de justificar a los stakeholders por qué existe tal discrepancia, siendo fruto de la inversión en recursos intangibles que genera ventaja competitivas en el futuro. En este sentido, Vergauwen et al., (2007) encuentran una asociación positiva y significativa entre el nivel de información del Capital Intelectual y el ratio market to book. Precisamente, se plantea la siguiente hipótesis:

- H5: El ratio market to book influye de manera positiva en la divulgación de información de Capital Intelectual. 
Además, hemos incluido el año como variable de control, siendo el número total de observaciones de 115 que proceden de las 23 empresas durante los cinco años de análisis.

\section{DISEÑO METODOLÓGICO Y OBTENCIÓN DE DATOS}

Para cuantificar la información del Capital Intelectual, Relacional, Estructural y Humano suministrada por las empresas se han elaborado índices de divulgación (Bukh et al., 2005; Cerbioni y Parbonetti, 2007; Firer y Williams, 2005; García-Meca et al., 2005) empleando la metodología del análisis de contenido.

Se ha garantizado la fiabilidad del análisis de contenido mediante un único codificador que ha sido objeto de formación durante un amplio período, y las decisiones de codificación han llegado a un nivel aceptable en la muestra piloto realizada (Guthrie et al., 2004).

\subsection{Periodo de estudio}

Con motivo de la aprobación en España de la ley 26/2003 de 17 de julio de transparencia informativa, se ha elegido un periodo de 5 años que comprenden desde el 2004 al 2008 (ambos inclusive); dejando, para posteriores trabajos, el periodo 2008-2012, tras la aprobación de la ley 16/2007, 4 de julio, de reforma y adaptación de la legislación mercantil en materia contable para su armonización internacional con base en la normativa de la Unión Europea, y también al coincidir, a raíz de su entrada en vigor, con una serie de fusiones de empresas cotizadas en Ibex 35 a lo largo de los años 2009 y 2010, que hubiera distorsionado la serie histórica de las seleccionadas.

\subsection{Definición de la población en cuestión y elección de una muestra adecuada}

La población está configurada por las empresas que cotizan en el mercado continuo español incluidas en el índice bursátil del Ibex 35 en 2008, ya que son susceptibles de una mayor transparencia informativa y una mejor accesibilidad a los documentos que se van a considerar.

Se opta por un diseño de la muestra dirigido o no probabilístico considerando las empresas incluidas en el índice en 2008, y que pertenecen al mismo durante el resto de años. La muestra está formada por 23 grupos empresariales, un $65,7 \%$ de la población de partida. Además, se encuentran caracterizados todos los sectores (ver Figura 1).

La muestra final de este estudio comprende a 23 empresas para cada uno de los 5 años. Por tanto, el número total de observaciones asciende a 115 correspondiente a los informes anuales de empresa-año $(23 \times 5)$. 
Figura 1

Número de empresas por sectores en la muestra y en la población

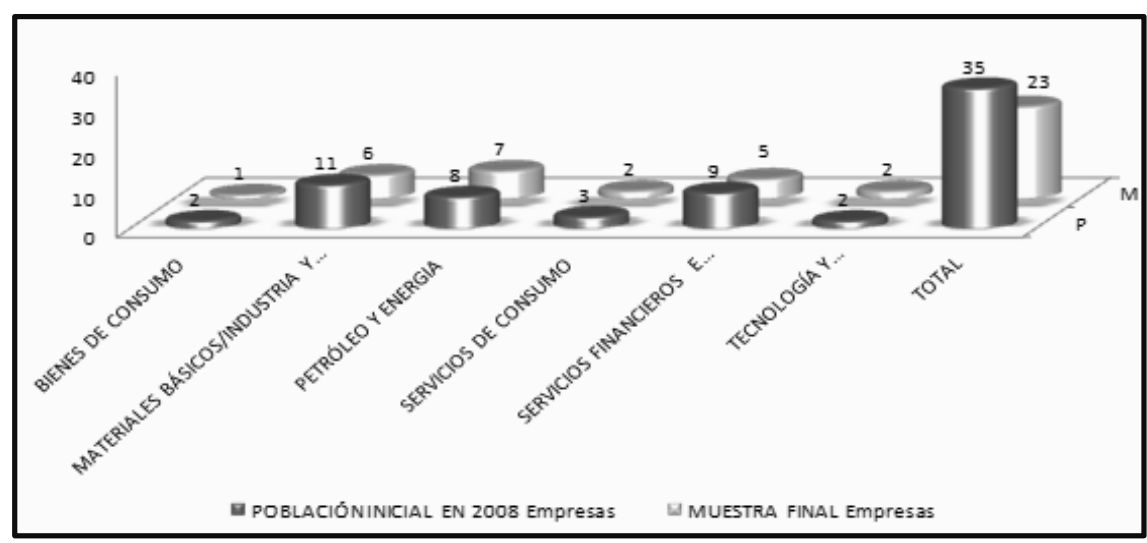

Fuente: Elaboración propia a partir de los informes publicados por Sociedad de Bolsas, SA.

\subsection{Definición de las variables y obtención de los datos}

\subsubsection{Variable dependiente}

El Capital Intelectual es la combinación de los recursos humanos (Capital Humano), organizativos (Capital Estructural) y relacionales (Capital Relacional). Por eso, el nivel de información sobre el Capital Intelectual, Relacional, Estructural y Humano suministrado por las empresas en sus informes o memorias anuales han sido las variables dependientes que se han considerado para los cuatro modelos que se especifican más adelante. Estas variables se han medido utilizando índices y subíndices de divulgación no ponderados.

\section{Tabla 1}

Categorías, subcategorías y elementos intangibles

\begin{tabular}{|c|c|}
\hline & CAPITAL INTELECTUAL \\
\hline CAPITAL HUMANO & $\begin{array}{l}\text { EMPLEADOS: } \\
\text { 1. } \\
\text { 2. } \text { Perfil del empleado } \\
\text { 3. Segualdad y diversidad } \\
\text { 4. } \quad \text { Relaciones laborales y actividad sindical } \\
\text { 5. Implicación de los trabajadores con la comunidad } \\
\text { 6. Reconocimiento de empleados } \\
\text { 7. Empleados destacados } \\
\text { 8. Compromiso de los empleados } \\
\text { 9. } \quad \text { Motivación de los empleados } \\
\text { 10. Comportamiento de los empleados } \\
\text { 11. Datos económicos } \\
\text { EDUCACIÓN: } \\
\text { 12. Educación reglada } \\
\text { 13. Cualificación profesional }\end{array}$ \\
\hline
\end{tabular}


Tabla 1 (continuación)

Categorías, subcategorías y elementos intangibles

\begin{tabular}{|c|c|}
\hline \multicolumn{2}{|r|}{ CAPITAL INTELECTUAL } \\
\hline & $\begin{array}{l}\text { FORMACIÓN Y DESARROLLO: } \\
14 . \quad \text { Formación de empleados } \\
\text { 15. Desarrollo de empleados }\end{array}$ \\
\hline $\begin{array}{l}\text { CAPITAL } \\
\text { HUMANO }\end{array}$ & $\begin{array}{ll}\text { CONOCIMIENTOS RELACIONADOS CON EL TRABAJO: } \\
\text { 16. } & \text { Know-how (saber hacer) } \\
\text { 17. Experiencia profesional } \\
\text { 18. Antigüedad de los expertos } \\
\text { 19. Rendimientos y resultados de altos directivos } \\
\text { 20. ESPÍRITU EMPRENDEDOR. }\end{array}$ \\
\hline \multirow{4}{*}{$\begin{array}{l}\text { CAPITAL } \\
\text { ESTRUCTURAL }\end{array}$} & $\begin{array}{ll}\text { PROPIEDAD INTELECTUAL: } \\
21 . & \text { Patentes } \\
\text { 22. } & \text { Copyrights } \\
\text { 23. Secretos comerciales } \\
\text { 24. } \text { Marcas comerciales } \\
\text { 25. FILOSOFIA DE GESTIÓN. } \\
\text { 26. } \text { CULTURA CORPORATIVA. }\end{array}$ \\
\hline & $\begin{array}{l}\text { PROCESOS TECNOLÓGICOS Y DE GESTIÓN: } \\
27 . \quad \text { Procesos de Gestión } \\
\text { 28. Procesos Tecnológicos }\end{array}$ \\
\hline & $\begin{array}{l}\frac{\boldsymbol{I}+\boldsymbol{D + \boldsymbol { i }}:}{29 .} \text { Investigación y Desarrollo } \\
\text { 30. Innovación }\end{array}$ \\
\hline & $\begin{array}{l}\text { SISTEMAS DE INFORMACIÓN Y DE REDES: } \\
31 . \quad \text { Sistemas de información } \\
\text { 32. Sistemas de redes }\end{array}$ \\
\hline \multirow{5}{*}{$\begin{array}{c}\text { CAPITAL } \\
\text { RELACIONAL }\end{array}$} & $\begin{array}{ll}\text { 33. } & \text { RELACIONES FINANCIERAS. } \\
\text { 34. } & \text { MARCAS. }\end{array}$ \\
\hline & $\begin{array}{ll}\text { CLIENTES: } \\
35 . & \text { Nombre de clientes relevantes } \\
\text { 36. } & \text { Lealtad de clientes } \\
\text { 37. } & \text { Satisfacción del cliente } \\
\text { 38. } & \text { Servicios y apoyo a clientes }\end{array}$ \\
\hline & $\begin{array}{l}\text { REPUTACIÓN/IMAGEN DE EMPRESA: } \\
39 . \quad \text { Nombre de la empresa } \\
\text { 40. Contratos favorables } \\
\text { 41. Medidas de protección del medioambiente. } \\
\text { 42. Patrocinio y mecenazgo } \\
\text { 43. Responsabilidad social/Acción social } \\
\text { 44. Gobierno de la empresa }\end{array}$ \\
\hline & $\begin{array}{l}\text { SOCIOS DEL NEGOCIO: } \\
45 . \quad \text { Colaboración con empresas } \\
46 . \quad \text { Acuerdos de franquicia } \\
47 . \quad \text { Acuerdos de licencia }\end{array}$ \\
\hline & $\begin{array}{ll}\text { OTRAS RELACIONES DE EMPRESA: } \\
48 . & \text { Relaciones con proveedores } \\
\text { 49. } & \text { Relaciones con las AAPP } \\
\text { 50. } & \text { Relaciones con centros de investigación } \\
\text { 51. } & \text { Relaciones con los medios de comunicación } \\
\text { 52. } & \text { Relaciones con otros grupos de interés } \\
53 . & \text { CANALES DE DISTRIBUCIÓN. }\end{array}$ \\
\hline
\end{tabular}

Fuente: Elaboración propia a partir del marco propuesto por Sveiby (1997) y modificado por Guthriey Petty (2000). 
Para la codificación de los datos nos hemos basado en el marco inicial de Sveiby (1997), y posteriormente modificado por Guthrie y Petty (2000). Así, hemos utilizado un modelo tripartito de Capital Intelectual donde cada categoría (Capital Humano, Capital Relacional y Capital Estructural) se compone de una serie de subcategorías y de elementos intangibles específicos basándonos en la literatura previa.

Se han elaborado tres subíndices, Capital Humano, Relacional y Estructural que han sido configurados de la siguiente manera:

$$
\begin{aligned}
& I C I_{j}=\frac{1}{53} \sum_{i=1}^{53} X_{i j} \\
& I C H_{j}=\frac{1}{20} \sum_{i=1}^{20} X_{i j} ; I C E_{j}=\frac{1}{12} \sum_{i=21}^{32} X_{i j} ; I C R_{j}=\frac{1}{21} \sum_{i=33}^{53} X_{i j}
\end{aligned}
$$

Donde $I j$ es el índice absoluto de divulgación no ponderado de la empresa $j$ ( $C I$, Capital Intelectual; $C H$, Capital Humano; $C E$, Capital Estructural y $C R$, Capital Relacional), $i$ son los ítems o partidas, $j$ es la empresa y, $X i j$ es la puntuación obtenida para el ítem $i$ en la empresa $j$. De modo que, $X i j$ tomará el valor 1 si la empresa $j$ ha divulgado el ítem $i$ y, tomará el valor 0 en caso contrario.

\subsubsection{Variables independientes y de control}

La obtención de los datos se ha realizado desde el informe o memoria anual y la base de datos SABI. La medición de estas variables queda descrita a continuación:

\section{Tabla 2}

Variables independientes y de control

\begin{tabular}{ll}
\hline \multicolumn{1}{c}{ VARIABLE } & \multicolumn{1}{c}{ MEDIDA } \\
\hline Tamaño & - Logaritmo neperiano de la capitalización bursátil \\
& - Logaritmo neperiano activos totales \\
& - Número de empleados. \\
\hline Sector & - Variable dicotómica (dummy), que recibe un valor igual a 1 si la empresa \\
& pertenece al sector en cuestión y un valor 0 si no pertenece \\
\hline Rentabilidad & - Rentabilidad Económica: ROA $\rightarrow$ Beneficio antes de Intereses e Impuestos/Activo \\
& Total \\
& - Rentabilidad Financiera: ROE $\rightarrow$ Beneficio después de Impuestos/Fondos Propios \\
\hline Endeudamiento & - Importe Total de la Deuda/Patrimonio Neto \\
\hline Market to Book & - Valor de capitalización/Valor contable del patrimonio neto al cierre del ejercicio \\
& contable \\
\hline Año & - Variable que tomará el valor de 1, para el año 2004, 2, para el 2005, 3, para el \\
& 2006, 4, para el 2007 y 5, para el año 2008 \\
\hline
\end{tabular}

Fuente: Elaboración propia. 


\subsubsection{Modelo de investigación}

Para obtener las variables que contribuyen, en mayor medida, a explicar la variabilidad del índice y subíndices de información de Capital Intelectual, se han utilizado cuatro modelos de regresión lineal múltiple que permiten, dada una variable dependiente a explicar y un conjunto de variables independientes, obtener una función lineal de tales variables con la cual explicar o predecir el valor de la variable dependiente. De esta manera, se crean los cuatro modelos siguientes:

- Modelo 1:

$$
I C I_{i t}=\alpha+\sum_{j=1}^{14} \beta_{j} \text { Variables de Características Empresariales }_{j i t}+\varepsilon
$$

- Modelo 2:

$$
I C R_{i t}=\alpha+\sum_{j=1}^{14} \beta_{j} \text { Variables de Características Empresariales }_{j i t}+\varepsilon
$$

- Modelo 3:

$$
I C E_{i t}=\alpha+\sum_{j=1}^{14} \beta_{j} \text { Variables de Características Empresariales }_{j i t}+\varepsilon
$$

\section{- Modelo 4:}

$$
I_{C H}=\alpha+\sum_{j=1}^{14} \beta_{j} \text { Variables de Características Empresariales }_{j i t}+\varepsilon
$$

Donde:

$I C I, I C R, I C E$ e $I C H=$ Índice de Capital Intelectual, Relacional, Estructural y Humano.

$i=1, \ldots . .23 ; t=1, \ldots .5$.

$j=$ las 14 variables relativas a las características de las empresas.

$B=$ Parámetro.

$\varepsilon=$ Término de error.

\section{ANÁLISIS DE DATOS Y RESULTADOS}

\subsection{Análisis descriptivo}

A continuación, en la Tabla 3, llevamos a cabo un análisis descriptivo para aproximarnos al comportamiento de las variables de nuestros modelos mediante sus principales estadísticos. 
Tabla 3

Estadísticos descriptivos de las variables

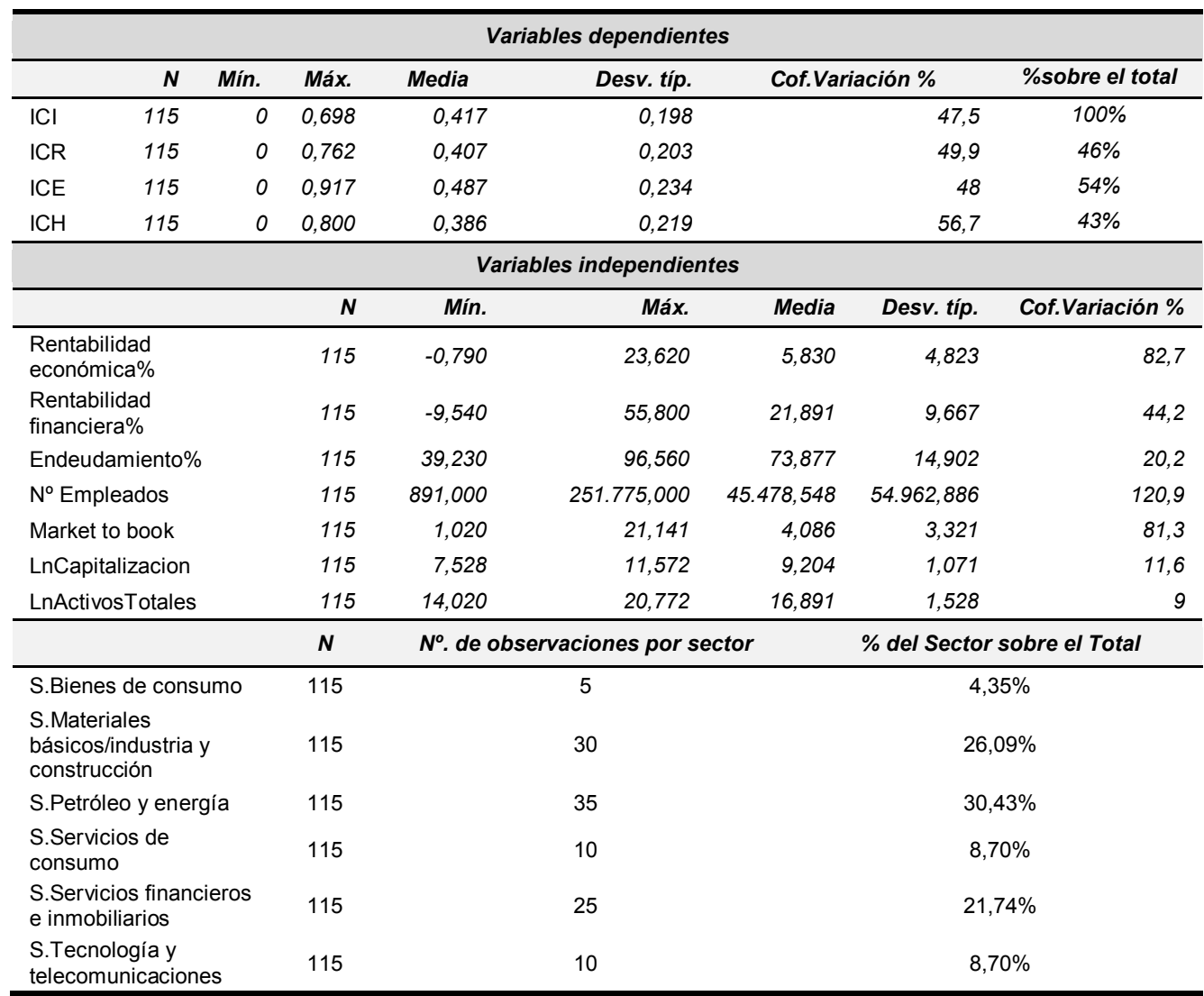

Fuente: Elaboración propia.

Los resultados del análisis descriptivo de nuestro estudio reflejan un porcentaje medio del nivel de divulgación del Capital Intelectual de un $42 \%$. Un valor todavía bajo, a pesar de que las empresas están inmersas en la Era del Conocimiento. Un motivo por el cual las empresas se sienten reticentes a suministrar información del Capital Intelectual puede deberse a que tienen miedo a sus competidores, como saben que es una de las principales fuentes de valor de la empresa y generadoras de ventajas competitivas, no quieren dar señales de esas ventajas a sus competidores.

Con relación a los porcentajes de participación en el Capital Intelectual de las tres categorías, Capital Relacional, Estructural y Humano, el porcentaje medio ha ascendido al $46 \%, 54 \%$ y $43 \%$, respectivamente. En la Tabla 4 se recoge una comparativa con estudios previos. En nuestro trabajo, la categoría menos 
divulgada ha sido la del Capital Humano. Este resultado es coincidente con la mayoría de los trabajos anteriores. Sin embargo, sorprende que para el caso de las empresas españolas pertenecientes al Ibex 35 , la categoría más divulgada ha sido la relativa al Capital Estructural para el periodo 2004-2008. Un resultado que refleja que lo que más divulgan las empresas es el conocimiento que permanece siempre dentro de ellas. También parece lógico pensar que, el Capital Humano sea la categoría menos divulgada puesto que no quieren enviar señales de un activo para la empresa tan valioso como es su Capital Humano, puesto que éste es el activo pensante y tiene decisión propia para poder abandonar la empresa.

Tabla 4

Resultados de otros estudios previos

\begin{tabular}{|c|c|c|c|c|}
\hline ESTUDIO & PAís & $\begin{array}{l}\text { CAPITAL } \\
\text { RELACIONAL }\end{array}$ & $\begin{array}{c}\text { CAPITAL } \\
\text { ESTRUCTURAL }\end{array}$ & $\begin{array}{l}\text { CAPITAL } \\
\text { HUMANO }\end{array}$ \\
\hline Este trabajo & España & $46 \%$ & $54 \%$ & $43 \%$ \\
\hline $\begin{array}{l}\text { Guthrie et al., (1999); } \\
\text { Guthrie y Petty (2000) }\end{array}$ & Australia & $40 \%$ & $30 \%$ & $30 \%$ \\
\hline Brennan (2001) & Irlanda & $40 \%$ & $30 \%$ & $30 \%$ \\
\hline April et al., (2003) & Sudáfrica & $40 \%$ & $30 \%$ & $30 \%$ \\
\hline Bozzolan et al., (2003) & Italia & $49 \%$ & $30 \%$ & $21 \%$ \\
\hline $\begin{array}{l}\text { Abeysekera y Guthrie } \\
(2005)\end{array}$ & Sri Lanka & $44 \%$ & $20 \%$ & $36 \%$ \\
\hline Goh y Lim (2004) & Malasia & $41 \%$ & $37 \%$ & $22 \%$ \\
\hline $\begin{array}{l}\text { Oliveras y Kasperskaya } \\
\text { (2005) }\end{array}$ & España & $51 \%$ & $28 \%$ & $21 \%$ \\
\hline Vandemaele et al., (2005) & $\begin{array}{l}\text { Suecia, Países } \\
\text { Bajos y Reino } \\
\text { Unido }\end{array}$ & $40 \%$ & $30 \%$ & $30 \%$ \\
\hline Wong y Gardner (2005) & Nueva Zelanda & $48 \%$ & $21 \%$ & $31 \%$ \\
\hline Monclús (2006) & España & $28,79 \%$ & $19,19 \%$ & $52,02 \%$ \\
\hline $\begin{array}{l}\text { Cerbioni y Parbonetti } \\
(2007)\end{array}$ & $\begin{array}{l}\text { Reino Unido, } \\
\text { Alemania y Suecia }\end{array}$ & $31,8 \%$ & $49,6 \%$ & $18,6 \%$ \\
\hline Steenkamp (2007) & Nueva Zelanda & $36 \%$ & $11 \%$ & $53 \%$ \\
\hline $\begin{array}{l}\text { Sujan y Abeysekera } \\
(2007)\end{array}$ & Australia & $48 \%$ & $31 \%$ & $21 \%$ \\
\hline Oliveras et al., (2008) & España & $60 \%$ & $18 \%$ & $22 \%$ \\
\hline Whitting y Miller (2008) & Nueva Zelanda & $47 \%$ & $20 \%$ & $33 \%$ \\
\hline Striukova et al., (2008) & Reino Unido & $61 \%$ & $17 \%$ & $22 \%$ \\
\hline Yi y Davey (2010) & China & $46 \%$ & $30 \%$ & $24 \%$ \\
\hline
\end{tabular}

Fuente: Elaboración propia.

Estos resultados sugieren la necesidad de un marco común y generalmente aceptado para poder realizar estudios comparativos con otros países. En esta tipología de estudios solamente se pueden comparar las categorías que son más 
divulgadas y las que menos, principalmente por varias razones: en primer lugar, el tamaño de la muestra es distinto; en segundo lugar, la metodología del análisis de contenido conlleva una gran subjetividad a la hora de codificar los datos y por último, el periodo objeto de análisis. Éstas serán las típicas limitaciones que presentan este tipo de trabajos.

\subsection{Resultados de la regresión múltiple lineal}

La estimación del modelo de regresión lineal se realiza mediante el método de regresión por etapas "hacia adelante", en el que la entrada y salida de las variables independientes en la ecuación de regresión se realiza a partir de los valores del estadístico t-Student. De tal manera que en los resultados sólo figuran las variables más significativas del modelo, es decir, aquellas que presentan una asociación significativa con la variable dependiente. Así, en cada paso se calcula el coeficiente estandarizado para cada variable si la misma entrase en el modelo en el próximo paso, así como el valor del estadístico t y el nivel de significación. En este trabajo, se ha utilizado como criterio de entrada en la ecuación un nivel de significación inferior al 0,05 y, uno de salida, superior al 0,1. A continuación, se exponen los resultados que se han obtenido en los cuatro modelos tras haberse cumplido los supuestos de linealidad, independencia, homocedasticidad, normalidad y no multicolinealidad para su correcta aplicación.

\section{Tabla 5}

Resultado del modelo de regresión lineal del Índice de Capital Intelectual

\begin{tabular}{|c|c|c|c|}
\hline \multirow{2}{*}{ Variables } & \multicolumn{3}{|c|}{$\begin{array}{c}\text { Modelo } 1 \\
\text { Índice Capital Intelectual } \\
\end{array}$} \\
\hline & $\beta$ & $\mathbf{t}$ & Sig. \\
\hline (Constante) & & 0,464 & 0,644 \\
\hline $\mathrm{N}^{\circ}$.Empleados & 0,172 & 1,945 & 0,054 \\
\hline Endeudamiento & 0,416 & 3,456 & 0,001 \\
\hline S.Servicios de consumo & $-0,302$ & $-3,577$ & 0,001 \\
\hline S.Servicios financieros e inmobiliarios & $-0,486$ & $-4,171$ & 0,000 \\
\hline$R^{2}$ & & & 0,264 \\
\hline$R^{2}$ corregido & & & 0,237 \\
\hline error típico & \multicolumn{3}{|c|}{0,173068404642} \\
\hline$F$ & \multicolumn{3}{|c|}{9,842} \\
\hline Sig $F$ & \multicolumn{3}{|r|}{0,000} \\
\hline
\end{tabular}

Fuente: Elaboración propia.

En la Tabla 5 se observa que son cuatro las variables que mejor contribuyen a explicar la cantidad de información de Capital Intelectual: a) el tamaño de la empresa resulta determinante, a mayor tamaño más información se divulga, así 
se reconoce tanto a nivel teórico ( $\mathrm{T}^{\mathrm{a}}$ de la Agencia, Señal, Legitimidad y Stakeholders) como empírico (Bozzolan et al., 2003; García-Meca et al., 2005; Guthrie et al., 2006, 2007); b) el nivel de endeudamiento también es un factor determinante del suministro de información (Archambault y Archambault, 2003; Hossain et al., 1995; Williams, 2001) con la intención de asegurar a los acreedores que la empresa tiene capacidad para devolver sus deudas $\left(\mathrm{T}^{\mathrm{a}}\right.$ de la Agencia y de la Señal); c) el sector de servicios de consumo y financiero e inmobiliario que mantienen comportamiento diferenciales respecto al resto de sectores, al ser sectores poco intensivos en intangibles (OECD, 2001), enviando pocas señales al mercado con relación a la información sobre el Capital Intelectual ya que no están interesadas en suministrar dicha información a sus stakeholders. Respecto a la bondad de este modelo, el R2 corregido asciende a 0,237 , lo que implica que las variables independientes explican un $23,7 \%$ de la varianza del nivel de divulgación de la información del Capital Intelectual. Este valor es muy similar al obtenido en el modelo de García-Meca et al., (2005) que asciende a $25,3 \%$, cuando analiza los informes de analistas financieros.

Por tanto, se confirman las hipótesis H1, H2 y H4. Sin embargo, la hipótesis H3 no ha resultado significativa en nuestro modelo, estos resultados son coincidentes con los obtenidos por Whiting y Woodcock (2011) y Ferreira et al., (2012). De la misma manera, la H5 tampoco ha resultado significativa para el caso del Capital Intelectual, un resultado coincidente con los trabajos de Brennan (2001), Whiting y Miller (2008) y Ferreira et al., (2012) que tampoco encontraron una relación significativa entre el market to book y el nivel de información del Capital Intelectual.

En el caso del Índice de Capital Relacional (ver Tabla 6), e igual que para el caso del Capital Intelectual (ver Tabla 5), el tamaño de la empresa contribuye a explicar los niveles de información. Además, el ratio market to book también es un factor determinante a la hora de suministrar información relativa al Capital Relacional, Vergauwen et al., (2007) encuentran también una asociación positiva con el ratio market to book. En nuestro caso, empresas con ratios elevados están más interesadas en suministrar información relativa al conocimiento que se genera de las relaciones con el entorno, puesto que están enviando señales a sus stakeholders acerca del motivo de la existencia de diferencias entre el valor de mercado y el contable que tiene su origen (en parte) en las buenas relaciones mantenidas con ellos, y desean seguir teniendo la aprobación de éstos para sobrevivir. Además, el endeudamiento no ha resultado significativo, a pesar de considerarse las relaciones financieras como uno de los principales intangibles dentro de esta categoría, una de las causas puede ser que las empresas no están interesadas en divulgar este tipo de información, puesto que ya cuentan con el apoyo de estos grupos de interés. Por otro lado, destaca que el sector del petróleo y la energía tiene una influencia significativa en la divulgación de información del Capital Relacional; uno de los motivos puede deberse a que es un sec- 
tor muy sensible al medioambiente y le interesa mantener buenas relaciones con su entorno socioeconómico para legitimar sus actuaciones. El R2 corregido asciende a 0,162 , por tanto, sólo explica un $16,2 \%$ de la varianza del nivel de información de Capital Relacional, a pesar de ser un valor bajo, el p-valor de la $\mathrm{F}$, que toma el valor de 0,000 , resulta significativo. Se corroboran las hipótesis $\mathrm{H} 1, \mathrm{H} 2$ y $\mathrm{H} 5$.

\section{Tabla 6}

Resultado de los modelos de regresión lineal de los subíndices de Capital Relacional,

Estructural y Humano

\begin{tabular}{|c|c|c|c|c|c|c|c|c|c|}
\hline \multirow[t]{2}{*}{ Variables } & \multicolumn{3}{|c|}{$\begin{array}{c}\text { Modelo } 2 \\
\text { Índice Capital } \\
\text { Relacional }\end{array}$} & \multicolumn{3}{|c|}{$\begin{array}{c}\text { Modelo } 3 \\
\text { Índice Capital } \\
\text { Estructural }\end{array}$} & \multicolumn{3}{|c|}{$\begin{array}{c}\text { Modelo } 4 \\
\text { Índice Capital Humano }\end{array}$} \\
\hline & $\beta$ & $\mathbf{t}$ & Sig. & $\beta$ & $t$ & Sig. & $\beta$ & $t$ & Sig. \\
\hline (Constante) & & 6,537 & 0,000 & & 0,403 & 0,688 & & $-0,969$ & 0,334 \\
\hline$N^{\circ}$.Empleados & 0,403 & 4,375 & 0,000 & 0,237 & 2,696 & 0,008 & & & \\
\hline Endeudamiento & & & & 0,405 & 3,374 & 0,001 & 0,535 & 4,790 & 0,000 \\
\hline Market to book & 0,236 & 2,667 & 0,009 & & & & & & \\
\hline $\begin{array}{l}\text { S.Servicios de } \\
\text { consumo }\end{array}$ & & & & $-0,219$ & $-2,599$ & 0,011 & $-0,373$ & $-4,555$ & 0,000 \\
\hline $\begin{array}{l}\text { S.Servicios financieros } \\
\text { e inmobiliarios }\end{array}$ & & & & $-0,507$ & $-4,374$ & 0,000 & $-0,522$ & $-4,623$ & 0,000 \\
\hline S.Petróleo y energia & 0,203 & 2,143 & 0,034 & & & & & & \\
\hline$R^{2}$ & & & 0,184 & & & 0,268 & & & 0,277 \\
\hline$R^{2}$ corregido & & & 0,162 & & & 0,242 & & & 0,258 \\
\hline error típico & & 0,1854 & 79485 & & 0,203351 & 149229 & & & 0,18900 \\
\hline$F$ & & & 8,363 & & & 10,083 & & & 14,210 \\
\hline Sig $F$ & & & 0,000 & & & 0,000 & & & 0,000 \\
\hline
\end{tabular}

Fuente: Elaboración propia.

Las variables que contribuyen a explicar el Índice de Capital Estructural (ver Tabla 6), y al igual que ocurría para el caso del Capital Intelectual, son: el tamaño de la empresa, el nivel de endeudamiento y los sectores de servicios de consumo y financiero e inmobiliario; como se ha comentado anteriormente, son sectores poco intensivos en intangibles y no están interesados en suministrar información sobre su Capital Estructural, puesto que no son las principales fuentes de riqueza para dichas empresas. El R2 corregido asciende a 0,242, explicando un $24,2 \%$ de la varianza del nivel de divulgación de la información del Capital Estructural. Las hipótesis H1, H2 y H4 son verificadas.

En el caso del Índice de Capital Humano (ver Tabla 6), las variables que inciden en el nivel de información del Capital Humano son las mismas que para el caso del índice del Capital Intelectual y Estructural, a excepción del tamaño de la empresa. Esto puede deberse a que hay una tendencia a suministrar informa- 
ción sobre el Capital Humano en los denominados informes de sostenibilidad o de responsabilidad social corporativo y, en nuestro caso analizamos exclusivamente los informes anuales. En este caso, el R2 corregido es el más elevado, explicando un $25,8 \%$ de la varianza del nivel de divulgación de la información del Capital Humano. Se confirman las hipótesis H2 y H4.

\section{CONCLUSIONES}

Este trabajo ha pretendido poner de manifiesto que, a pesar de la estricta normativa contable en cuanto al tratamiento de los recursos intangibles/Capital Intelectual, las empresas españolas pertenecientes al índice bursátil del Ibex 35 están suministrando de manera voluntaria información sobre el Capital Intelectual. Aunque las empresas se encuentran inmersas en la Era del Conocimiento, los niveles de información suministrados por éstas son bajos.

Además, al igual que ha ocurrido en estudios realizados en otros países, la categoría perteneciente al Capital Humano ha sido la menos divulgada por las empresas españolas. Sin embargo, al contrario de los resultados de trabajos previos, el Capital Estructural ha sido la categoría más revelada en los informes anuales. Ello puede deberse a dos motivos: en primer lugar, puede suceder que las empresas estén interesadas en suministrar aquel conocimiento que siempre permanece en la empresa por miedo a revelar otros recursos intangibles que pueden favorecer a la competencia; y en segundo lugar, puede suceder que exista una tendencia a suministrar información del Capital Humano y del Capital Relacional en otros informes o memorias distinta al informe anual, tal es el caso de las memorias de sostenibilidad o de responsabilidad social corporativa.

Por otro lado, y al igual que ha ocurrido en otros trabajos realizados para empresas de distintos países (Bozzolan et al., 2003; Guthrie et al., 2006, 2007), existen determinadas características empresariales que condicionan el mayor/menor suministro de información relativa al Capital Intelectual.

Así, hemos contrastado que son las empresas de mayor tamaño las que suministran mayor nivel de información del Capital Intelectual (Bozzolan et al., 2003; García-Meca et al., 2005; Guthrie et al., 2006, 2007), Relacional y Estructural con la finalidad de reducir las posibles asimetrías informativas y los costes de agencia. Además, al ser más grandes están más expuestas a la opinión pública y, por tanto, existe un mayor número de stakeholders interesados en ellas, por lo que suministran más información voluntaria al respecto. Lo cual confirma la primera hipótesis de partida.

Igualmente, señalar que en línea con otros estudios empíricos (Bozzolan et al., 2003; García-Meca et al., 2005), el efecto del sector sobre el nivel de divulgación también ha resultado significativo, corroborándose la hipótesis segunda. 
Además, se ha corroborado la hipótesis cuarta, contribuyendo el nivel de endeudamiento a explicar el mayor nivel de divulgación del Capital Intelectual, Estructural y Humano, al igual que sucedía en el trabajo de Williams (2001), con la finalidad de enviar señales a los acreedores de que la empresa ha invertido en intangibles y tiene capacidad para devolver sus deudas, minorando también los costes de agencia.

De igual manera, se confirma la quinta hipótesis, al existir una relación positiva y significativa entre el ratio market to book y el suministro de información del Capital Relacional, que también ha sido sugerido en otros estudios (Vergauwen et al., 2007).

Por último, añadir que la tercera hipótesis no ha podido ser avalada en este trabajo empírico, al igual que ocurría en el estudio de García Meca et al., (2005), que no podían confirmar la influencia positiva de la rentabilidad en la divulgación de información de Capital Intelectual a través de las presentaciones a analistas.

Sin embargo, este estudio tiene algunas limitaciones. En primer lugar, el tamaño de la muestra es pequeño y limitado a una muestra de empresas españolas con unos niveles de capitalización bursátil elevados, esto supone que no se pueda generalizar para todo el contexto de empresas españolas, si bien investigaciones futuras podrían ir encaminadas a superar dichas limitaciones. Y, en segundo lugar, este estudio no abarca la totalidad de medios de los que dispone la empresa para suministrar información de carácter voluntaria, exclusivamente nos hemos centrado en uno de los más relevantes, el informe anual; si bien, en futuros trabajos se podrían considerar otras vías de comunicación, como páginas web y los informes de responsabilidad social corporativa.

Nuestros resultados pretenden ser útiles y ayudar a organismos e instituciones, públicos y privados, a intentar elaborar unas guías o directrices respecto a la presentación de información voluntaria del Capital Intelectual, o bien, a desarrollar mejores normativas contables, ya que se ha evidenciado que con la finalidad de minorar la brecha existente entre el valor de mercado y el contable, las empresas sí están suministrando información adicional a sus grupos de interés con el propósito de que éstos tomen decisiones eficientes.

Esto puede ser especialmente relevante para el resto del tejido empresarial en cuanto a mejorar los niveles y la cantidad de información suministrada del Capital Intelectual, puesto que las empresas (bajo los postulados de diferentes teorías: agencia, señal, stakeholders, legitimidad y recursos y capacidades) son conscientes de la importancia que tiene el Capital Intelectual para sus grupos de interés y se dan a conocer sólo por algunas empresas. 


\section{REFERENCIAS BIBLIOGRÁFICAS}

ABEYSEKERA, I. (2006). "The project of intellectual capital disclosure: Resear-ching the research". Journal of Intellectual Capital, 7(1), pp. 61-75.

ABEYSEKERA, I. (2007). "Intellectual capital reporting between a developing and developed nation". Journal of Intellectual Capital, 8(2), pp. 329-345.

ABEYSEKERA, I. y GUTHRIE, J. (2005). "An empirical investigation of annual reporting trends of intellectual capital in Sri Lanka". Critical Perspectives on Accounting, 16(3), pp. 151-163.

APRIL, K.A.; BOSMA, P. y DEGLON, D.A. (2003). "IC measurement and repor-ting: Establishing a practice in SA mining". Journal of Intellectual Capital, 4(2), pp. 165180.

ARCHAMBAULT, J.J. y ARCHAMBAULT, M.E. (2003). "A multinational test of determinants of corporate disclosure". International Journal of Accounting, 38(2), pp. 173-194.

BARAKO, D.G., HANCOCK, P. y IZAN, H.Y. (2006). "Relationship between corporate governance attributes and voluntary disclosures in annual reports: the Kenyan experience". Financial Reporting Regulation and Governance, 5(1), pp. 1-27.

BEATTIE, V. (2005). "Moving the financial accounting research front forward: The UK contribution". British Accounting Review 37(1), pp. 85-114.

BEAULIEU, P.R., WILLIAMS, S.M. y WRIGHT, M.E. (2002). "Intellectual capital disclosures in Swedish annual reports", en: Bontis, N.E. (Ed.), World Con-gress on Intellectual Capital Readings. Butterworth-Heinemann, Hamilton, pp. 135-156.

BELKAOUI, A.R. y KARPIK, P.G. (1989). "Determinants of the corporate decision to disclose social information". Accounting, Auditing and Accounta-bility Journal, 2(1), pp. 36-51.

BONSÓN, E. y ESCOBAR, T. (2004). "La difusión voluntaria de información financiera en Internet. Un análisis comparativo entre Estados Unidos, Euro-pa del Este y la Unión Europea”. Revista Española de Financiación y Conta-bilidad, XXXIII(123), pp. 1063-1101.

BONTIS, N. (2003). "Intellectual capital disclosure in Canadian corporations". Journal of Human Resource Costing and Accounting, 7(1), pp. 9-20.

BOZZOLAN, S., FAVOTTO, F. y RICCERI, F. (2003). "Italian annual intellectual capital disclosure: An empirical analysis". Journal of Intellectual Capital, 4(4), pp. 543-558.

BRANCO, M.C., DELGADO, C., SOUSA, C. y SÁ, M. (2011). "Intellectual capi-tal disclosure media in Portugal". Corporate Communications: An Internatio-nal Journal, 16(1), pp. 38-52.

BRENNAN, N. (2001). "Reporting intellectual capital in annual reports: Evidence from Ireland". Accounting, Auditing \& Accountability Journal, 14(4), pp. 423-436.

BROOKING, A. (1997). El capital intelectual: el principal activo de las empresas del tercer milenio. Paidós Empresa, Barcelona.

BUENO, E., SALMADOR, M.P. y MERINO, C. (2008). "Génesis, concepto y desarrollo del capital intelectual en la economía del conocimiento: Una reflexión sobre el modelo Intellectus y sus aplicaciones". Estudios de Econo-mía Aplicada, 26(2), p. 3. 
BUKH, P.N. y JOHANSON, U. (2003). "Research and knowledge interaction: Guidelines for intellectual capital reporting". Journal of Intellectual capital, 4(4), pp. 576-587.

BUKH, P.N., NIELSEN, C., GORMSEN, P. y MOURITSEN, J. (2005). "Disclosure of information on intellectual capital in Danish IPO prospectu-ses". Accounting, Auditing and Accountability Journal, 18(6), pp. 713-732.

CAMPBELL, D.J. y ABDUL RAHMAN, M.R. (2010). "A longitudinal examination of intellectual capital reporting in Marks \& Spencer annual reports, 1978-2008". British Accounting Review, 42(1), pp. 56-70.

CASTILLA, F. y GALLARDO, D. (2008). "La decisión de divulgar intangibles a través de una estrategia de triangulación teórica". Estudios de Economía Aplicada, 26(2), p. 5.

CAÑIBANO, L. y SÁNCHEZ, M.P. (2008). "Intellectual Capital Management and Reporting in Universities and Research Institutions". Estudios de Economía Aplicada, 26(2), p. 1.

CE, COMISIÓN EUROPEA (2006). Reporting intellectual capital to augment research, development and innovation in SMEs: report to the Commission of the High Level Expert Group on RICARDIS, Comisión Europea. Dirección General de Investigación ed. Oficina de Publicaciones Oficiales de las Co-munidades Europeas, Luxemburgo.

CERBIONI, F. y PARBONETTI, A. (2007). "Exploring the Effects of Corporate Governance on Intellectual Capital Disclosure: An Analysis of European Biotechnology Companies". European Accounting Review, 16(4), pp. 791-826.

CHANDER, S. y MEHRA, V. (2011). "A study on intangible assets disclosure: An evidence from Indian companies". Intangible Capital, 7(1), pp. 1-30.

COLLINS, D.W., MAYDEW, E.L. y WEISS, I.S. (1997). "Changes in the value-relevance of earnings and book values over the past forty years". Journal of Accounting and Economics, 24(1), pp. 39-67.

ENG, L.L. y MAK, Y.T. (2003). "Corporate governance and voluntary disclo-sure". Journal of Accounting and Public Policy, 22(4), pp. 325-345.

ENSSLIN, S.R. y DE CARVALHO, F.N. (2007). "Voluntary disclosure of inte-llectual capital in the Brazilian context: an investigation informed by the inter-national context. International". Journal of Accounting, Auditing and Performance Evaluation, 4(4-5), pp. 478-500.

EUROFORUM, (1998). Medición del capital intelectual: Modelo intelect. Institu-to Universitario Euroforum Escorial, Madrid.

FASB, FINANCIAL ACCOUNTING STANDARDS BOARD (2001a). Improving business reporting: Insights into enhancing voluntary disclosures. Steering Committee Report, Business reporting research project. Financial Accoun-ting Standard Board.

FASB, FINANCIAL ACCOUNTING STANDARDS BOARD (2001b). Statement No.142, Goodwill and other intangible assets. Financial Accounting Stan-dards Board.

FERREIRA, A.L., BRANCO, M.C. y MOREIRA, J.A. (2012). "Factors influencing intellectual capital disclosure by Portuguese companies". International Jour-nal of Accounting and Financial Reporting, 2(2), pp. 278-298.

FIRER, S. y WILLIAMS, S.M. (2005). "Firm ownership structure and intellectual capital disclosures". Journal of Accounting Research, 19(1), pp. 1-18.

FRANCIS, J. y SCHIPPER, K. (1999). "Have financial statements lost their rele-vance?". Journal of Accounting Research, 37(2), pp. 319-352.

FREEMAN, R.E. (1984). Strategic Management: A Stakeholder Approach. Pit-man Publishing, Boston, MA. 
GAN, K., SALEH, Z. y ABESSI, M. (2008). Corporate Governance, Ownership Structures and Intellectual Capital Disclosures: Malaysian Evidence. 16th Conference on the Theories and Practices of Securities and Finance Mar-kets. Department of Finance, National Sun Yat-Sen University., 5-6 Diciem-bre 2008, Kaohsiung, Taiwan.

GANDÍA CABEDO, J.L. (2002). "La divulgación de información sobre intangibles en Internet: evidencia internacional". Revista Española de Financiación y Contabilidad, XXXI(113), pp. 767-802.

GANDÍA CABEDO, J.L. (2003). "Intangibles Disclosure Information on Internet by Multinational Corporations". The International Journal of Digital Accoun-ting Research, 3(5), pp. 61-99.

GARCÍA-AYUSO, M. (2003). "Factors explaining the inefficient valuation of intangibles". Accounting, Auditing \& Accountability Journal, 16(1), pp. 57-69.

GARCÍA-AYUSO, M., MONTERREY, J.A. y PINEDA, C. (1998). A Comparative Analysis of the Value Relevance of Accounting Information in the Capital Markets of the European Union, working paper. Universidad de Sevilla, Sevilla.

GARCÍA-MECA, E., PARRA, I., LARRÁN, M. y MARTÍNEZ, I. (2005). "The explanatory factors of intellectual capital disclosure to financial analysts". European Accounting Review, 14(1), pp. 63-94.

GINER, B. (1997). "The influence of company characteristics and accounting regulation on information disclosed by Spanish firms". European Accounting Review, 6(1), pp. 45-68.

GOH, P.C. y LIM, K.P. (2004). "Disclosing intellectual capital in company annual reports: Evidence from Malaysia". Journal of Intellectual Capital, 5(3), pp. 500-510.

GU, F. y LEV, B. (2001). Intangible assets: measurement, drivers, usefulness. working paper, Boston University, Boston, MA,.

GUTHRIE, J. y PETTY, R. (2000). "Intellectual capital: Australian annual reporting practices". Journal of Intellectual Capital, 1(3), pp. 241-251.

GUTHRIE, J., PETTY, R., FERRIER, F. y WELLS, R. (1999). There is no accounting for intellectual capital in Australia: A review of annual reporting practices and the internal measurement of intangibles, OECD Symposium on Measuring and Reporting of Intellectual Capital, Amsterdam.

GUTHRIE, J., PETTY, R. y RICCERI, F. (2006). "The voluntary reporting of intellectual capital: Comparing evidence from Hong Kong and Australia". Journal of Intellectual Capital, 7(2), pp. 254-271.

GUTHRIE, J., PETTY, R. y RICCERI, F. (2007). Intellectual Capital Reporting: Lessons from Hong Kong and Australia. Institute of Chartered Accountants of Scotland, Edinburgh.

GUTHRIE, J., PETTY, R., YONGVANICH, K. y RICCERI, F. (2004). "Using content analysis as a research method to inquire into intellectual capital reporting". Journal of Intellectual Capital, 5(2), pp. 282-293.

HALL, R. (1992). "The strategic analysis of intangible resources". Strategic Management Journal, 13(2), pp. 135-144.

HANIFFA, R.M. y COOKE, T.E. (2005). "The impact of culture and governance on corporate social reporting". Journal of Accounting and Public Policy, 24(5), pp. 391430. 
HOSSAIN, M., PERERA, M.H.B. y RAHMAN, A.R. (1995). "Voluntary disclosure in the annual reports of New Zealand companies". Journal of International Financial Management and Accounting, 6(1), pp. 69-87.

IASB, INTERNATIONAL ACCOUNTING STANDARDS BOARD (2004). Activos Intangibles, Normas Internacionales de Contabilidad, NIC 38. IASB, Lon-dres.

JENSEN, M.C. y MECKLING, W.H. (1976). "Theory of the firm: Managerial be-havior, agency costs and ownership structure". Journal of Financial Econo-mics, 3(4), pp. 305-360.

JOSHI, M., UBHA, D.S. y SIDHU, J. (2012). "Intellectual capital disclosures by Indian and Australian information technology companies: A comparative analysis". Journal of Intellectual Capital, 13(4), pp. 582-598.

KAPLAN, R.S. y NORTON, D.P. (1992). "The balanced scorecard--measures that drive performance". Harvard Business Review, 70(1), pp. 71-79.

KRIPPENDORFF, K. (1997). Metodología de análisis de contenido. Teoría y práctica. Paidós Comunicación, Barcelona.

LEV, B. (2003). Intangibles: medición, gestión e información. Deusto, Barce-lona.

LEV, B. y ZAROWIN, P. (1999). "The boundaries of financial reporting and how to extend them". Journal of Accounting Research, 37(2), pp. 353-385.

LI, J., PIKE, R. y HANIFFA, R. (2008). "Intellectual capital disclosure and corporate governance structure in UK firms". Accounting and Business Re-search, 38(2), pp. 137-159.

MARSTON, C.L. y SHRIVES, P.J. (1991). "The use of disclosure indices in accounting research: A review article". British Accounting Review, 23(3), 195-210.

MONCLÚS, R., RODRÍGUEZ, A. y TORRES, T. (2006). Información sobre intangibles en la nueva economía. Un estudio sobre las prácticas de las em-presas españolas cotizadas. Instituto de Análisis de Intangibles y ACCID, Barcelona.

OECD, ORGANISATION FOR ECONOMIC CO-OPERATION AND DEVELOPMENT (2001). OECD Science, Technology and Industry Sco-reboard 2001: Towards a Knowledge-based Economy. OECD Publishing, Paris.

OLIVEIRA, L., RODRIGUES, L.L. y CRAIG, R. (2010). "Intellectual capital reporting in sustainability reports". Journal of Intellectual Capital, 11(4), pp. 575-594.

OLIVERAS, E., GOWTHORPE, C., KASPERSKAYA, Y. y PERRAMON, J. (2008). "Reporting intellectual capital in Spain". Corporate Communications: An International Journal, 13(2), pp. 168-181.

OLIVERAS, E. y KASPERSKAYA, Y. (2005). Reporting Intellectual Capital in Spain, Economics and Business Working Papers Series 781. Universidad Pompeu Fabra, Barcelona.

PATTEN, D.M. (1991). "Exposure, legitimacy, and social disclosure". Journal of Accounting and Public Policy, 10(4), pp. 297-308.

PETTY, R. y CUGANESAN, S. (2005). "Voluntary disclosure of intellectual capital by Hong Kong companies: examining size, industry and growth effects over time". Australian Accounting Review, 15(36), pp. 40-50.

PGC, PLAN GENERAL DE CONTABILIDAD (2007). Real Decreto 1514/2007, de 16 de noviembre, por el que se aprueba el Plan General de Contabilidad. Suplemento del BOE $n^{\circ} 278,20$ de noviembre de 2007, Ministerio de Economía y Hacienda. 
PIZARRO, M.I., FERNÁNDEZ, R., CARLOS, J. y NAVARRO, R. (2011). "La incidencia del capital humano y la cultura emprendedora en la innovación". Cuadernos de Economía y Dirección de la Empresa, 14(3), pp. 139-150.

PULIDO, A. (2008). "Una revisión de conjunto de la economía de los intangibles". Estudios de Economía Aplicada, 26(2), pp. 29-42.

RAMíREZ, Y. (2010). "Análisis de las principales guías para la presentación de información sobre capital intelectual”. Estudios de Economía Aplicada, 28(2), p. 481.

RAMíREZ, Y., SANTOS, J.F. y TEJADA, A. (2011). "Beneficios y costes de la divulgación de información sobre capital intelectual: Evidencia empírica en las universidades públicas españolas". Estudios de Economía Aplicada, 29(3), pp. 1328.

ROSS, S.A. (1979). Disclosure Regulation in the Financial Markets: Implications of Modern Finance Theory and Signalling Theory. Mc Graw Hill, New York.

SHOCKER, A.D. y SETHI, S.P. (1973). "An approach to developing societal preferences in developing corporate action strategies“. California Manage-ment Review, XV(4), pp. 97-105.

SIMÓ, P. y SALLÁN, J.M. (2008). "Capital intangible y capital intelectual: Re-visión, definiciones y líneas de investigación". Estudios de Economía Aplica-da, 26(2), pp. 63-78.

SINGH, S. y KANSAL, M. (2011). "Voluntary disclosures of intellectual capital: an empirical analysis". Journal of Intellectual Capital, 12(2), pp. 301-318.

SINGHVI, S.S. y DESAI, H.B. (1971). "An empirical analysis of the quality of corporate financial disclosure". The Accounting Review, 46(1), pp. 129-138.

SONNIER, B.M. (2008). "Intellectual capital disclosure: high-tech versus traditional sector companies". Journal of Intellectual Capital, 9(4), pp. 705-722.

STEENKAMP, N. (2007). Intellectual capital reporting in New Zealand: refining content analysis as a research method. PhD thesis. Auckland University of Technology, Auckland (New Zealand).

STRIUKOVA, L., UNERMAN, J. y GUTHRIE, J. (2008). "Corporate reporting of intellectual capital: Evidence from UK companies". The British Accounting Review, 40(4), pp. 297-313.

SUJAN, A. y ABEYSEKERA, I. (2007). "Intellectual Capital reporting practices of the top Australian firms". Australian Accounting Review, 17(42), pp. 62-74.

SVEIBY, K.E. (1997). "The intangible assets monitor". Journal of Human Resource Costing and Accounting, 2(1), pp. 73-97.

SVEIBY, K.E. (2000). Capital intelectual. La nueva riqueza de las empresas: cómo medir y gestionar los activos intangibles para crear valor. Gestión 2000, Barcelona.

TAGESSON, T., BLANK, V.B., P. y COLLIN, S. (2009). "What explains the extent and content of social and environmental disclosures on corporate websites: a study of social and environmental reporting in Swedish listed corporations". Corporate Social Responsibility and Environmental Manage-ment, 16(6), pp. 352-364.

UPTON, W.S. (2001). Business and financial reporting, challenges from the new economy. Financial Accounting Standards Board of the Financial Ac-counting Foundation.

VANDEMAELE, S.N., VERGAUWEN, P.G.M.C. y SMITS, A.J. (2005). "Intellectual capital disclosure in The Netherlands, Sweden and the UK: A longitudinal and comparative study". Journal of Intellectual Capital, 6(3), pp. 417-426. 
VERGAUWEN, P.G.M.C., BOLLEN, L. y OIRBANS, E. (2007). "Intellectual capital disclosure and intangible value drivers: An empirical study". Management Decision, 45(7), pp. 1163-1180.

WAGICIENGO, M.M. y BELAL, A.R. (2012). "Intellectual capital disclosures by South African companies: A longitudinal investigation”. Advances in Accounting, 28(1), pp. 111-119.

WALLMAN, S. (1995). "The future of accounting and disclosure in an evolving world: the need for a dramatic change". Accounting Horizons, 9(3), pp. 81-91.

WEBER, R.P. (1990). Basic content analysis. SAGE, Newbury Park.

WHITE, G., LEE, A. y TOWER, G. (2007). "Drivers of voluntary intellectual capital disclosure in listed biotechnology companies". Journal of Intellectual Capital, 8(3), pp. 517-537.

WHITING, R.H. y MILLER, J.C. (2008). "Voluntary disclosure of intellectual capital in New Zealand annual reports and the hidden value". Journal of Hu-man Resource Costing \& Accounting, 12(1), pp. 26-50.

WHITING, R.H. y WOODCOCK, J. (2011). "Firm characteristics and intellectual capital disclosure by Australian companies". Journal of Human Resource Costing \& Accounting, 15(2), pp. 102-126.

WILLIAMS, S.M. (2001). "Is intellectual capital performance and disclosure practices related?". Journal of Intellectual Capital, 2(3), pp. 192-203.

WONG, M. y GARDNER, C.T. (2005). Intellectual capital disclosure: New Zea-land evidence, Conference paper presented at the AFFANZ 2005 conferen-ce, Melbourne, Australia.

YI, A. y DAVEY, H. (2010). "Intellectual capital disclosure in Chinese (mainland) companies". Journal of intellectual capital, 11(3), pp. 326-347. 
